\title{
Resolution of the ethanolamine salt of $( \pm$ )mandelic acid by using the AS3PC method: Principle, application and results
}

\author{
E. Aubin, M.N. Petit and G. Coquerel \\ UC ${ }^{2} M^{2}$, UPRES EA 3233, Université de Rouen, 76821 Mont Saint-Aignan, France \\ e-mail: gerard.coquerel@univ-rouen.fr
}

\begin{abstract}
The ethanolamine salt of $( \pm)$ mandelic acid crystallizes as a stable conglomerate. This study aims at determining if the racemic mixture could be efficiently resolved by preferential crystallization in the azeotropic mixture: Ethanol - Water at ca. $20^{\circ} \mathrm{C}$. Despite the optimisation of several pertinent parameters, the efficiency of the process and the optical purity of the crude crops have been found to remain low (e.e. fmax $_{\text {max }} \mid 5-7 \%$ with $\mid 65 \%$ e.e. purity (e.e. stands for enantiomeric excess)). In order to determine the origin of this poor entrainment effect precise XRPD measurements (on pure enantiomer and racemic mixture) coupled with DSC analyses have been undertaken: the results clearly show the existence of a partial solid solution whose maximal composition is approximately $36 \%$ e.e. at $106^{\circ} \mathrm{C}$ and probably below $10 \%$ e.e. at room temperature. Nevertheless the metastable domains of solid solution and/or the high supersaturation created must be also taken into consideration to explain the poor efficiencies.
\end{abstract}

\section{INTRODUCTION}

Beside the classical pasteurian resolution method, the preferential crystallization [1,3] presents many advantages such as: no need for any resolving agent and a quantitative yield (by means of successive recycling of the mother liquor). Nevertheless the applicability of this technique remains restricted to a

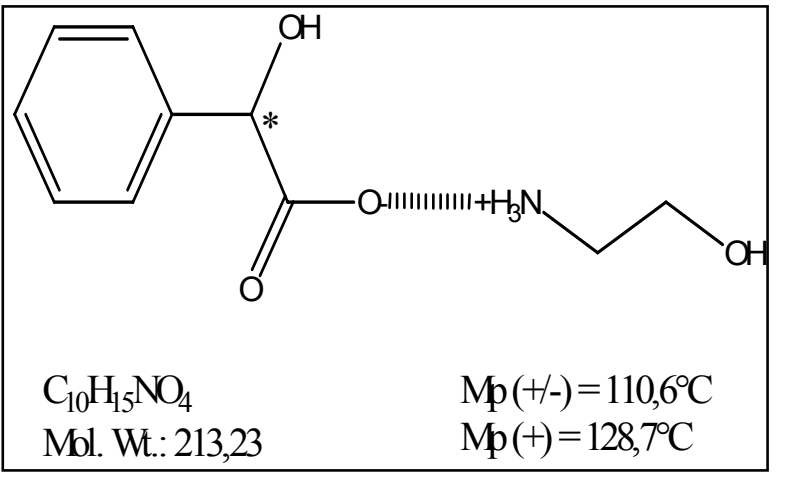

Figure 1. Ethanolamine salt of mandelic acid. small fraction of racemic mixtures which crystallize as stable conglomerates. Moreover, among the set of derivatives crystallizing as conglomerates only one half could be resolved with an acceptable efficiency [1]. This study aimed at determining if the ethanolamine salt of $(r)$ mandelic acid could be efficiently resolved by using the variant called AS3PC [1] (AS3PC is the acronym for Auto Seeded Programmed Polythermic Preferential Crystallization.) and if not to highlight the main factors that are responsible for the poor efficiency. 


\section{AS3PC PROCESS [3]}

- Starting with a racemic mixture (conglomerate) and an appropriate amount of solvent, a small amount $\mathrm{M}$ of pure enantiomer is added for the very first run (usually about $6 \%$ e.e. ${ }^{*}$, here we consider the $\mathrm{R}$ enantiomer).

$\rightarrow$ - The starting temperature $T_{B}$ is chosen so that only the opposite enantiomer (S) is completely dissolved.

- Then, the temperature is progressively decreased (following an adapted cooling program) in order to induce an enantioselective secondary nucleation and crystal growth of the enantiomer $(\mathrm{R})$ present in the initial suspension.

- Due to the entrainment effect it is possible to maintain the crystallization of the (R) enantiomer beyond the thermodynamic limit (e.e. $=0$ ); simultaneously the counter enantiomer remains supersaturated (i.e. no spontaneous nucleation of the opposite enantiomer (S) happens for a given period of time). The slurry must be filtered prior to the primary nucleation and growth of the $(\mathrm{S})$ enantiomer; at least $2 \mathrm{M}$ mass of pure crystals should ideally be collected. The mother liquor is now enriched in (S) enantiomer.

- A $2 \mathrm{M}$ mass of racemic mixture is added to the solution. It is possible to operate the selective crystallization of the $(S)$ enantiomer by reproducing the same sequence of operations ( $\mathrm{S}$ substituting for $\mathrm{R}$ and $\mathrm{R}$ for $\mathrm{S}$ ).

- By means of continuous recycling of the mother liquors, every odd operation gives the (R) enantiomer and every even crystallization gives the $(\mathrm{S})$ enantiomer. In terms, any amount of racemic mixture can be quantitatively resolved by using this process.

\section{EXPERIMENTS}

Routine XRPD measurements on the racemic mixture and on the pure enantiomer (cf. Figure 2) reveal at first sight the existence of a conglomerate. In order to test the efficiency of the preferential

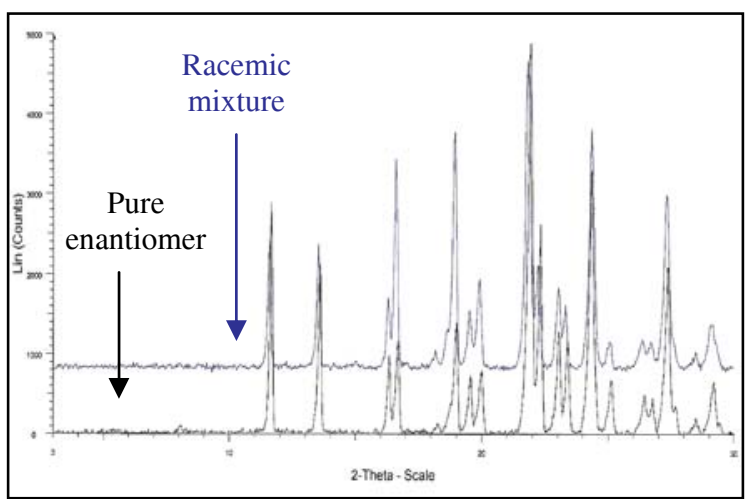

Figure 2. XRPD diffractograms of the ethanolamine mandelates: racemic mixture and pure enantiomer.

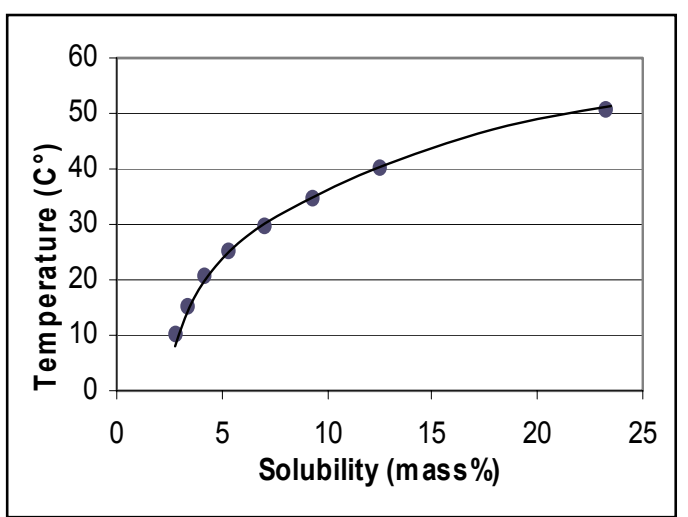

Figure 3. Solubility vs. temperature of $( \pm)$ ethanolamine mandelate in $\mathrm{EtOH}(96) / \mathrm{H}_{2} \mathrm{O}(4)$.

(*) e.e. stands for enantiomeric excess. 
crystallization the adjustment of several thermodynamic and kinetic parameters has to be performed. In a first step, a screening of solvents revealed that the azeotropic mixture ethanol/water (96/4 wt \%) gives suitable solubilities with $( \pm$ )ethanolamine mandelate (cf. Figure 3 ). These solubilities were determined by using the usual gravimetric method. At $40^{\circ} \mathrm{C}$ the solubility $(12.5 \%)$ is adapted for a productive yield and the viscosity of the slurry remains low enough for an easy and fast filtration.

In a second step, the metastable zone of crystallization has been evaluated: these data are essential to set up the cooling program. The goal of these experiments is to determine the domain in composition in which a sufficient supersaturation can be created for the existing (R) crystals without inducing a too fast nucleation of the counter enantiomer $(S)$ for a given time. Assessments have shown that a saturated solution at $40^{\circ} \mathrm{C}$ could be quenched at ca. $20^{\circ} \mathrm{C}$ without inducing the nucleation of the counter enantiomer for several tens of minutes.

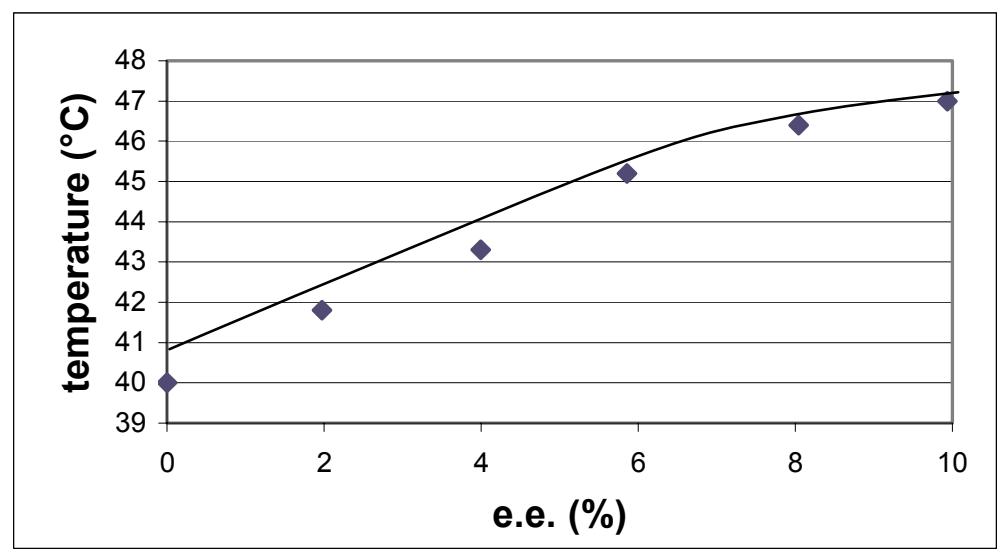

Figure 4. Homogeneisation temperature vs. enantiomeric excess.

The final step of the tuning is the determination for a given racemic salt concentration of the homogenisation temperature versus the enantiomeric excess. Experimentally, successive amounts of pure enantiomer were added to the saturated racemic solution $\left(\right.$ at $40^{\circ} \mathrm{C}$ ) and temperature was slowly increased up to the complete dissolution of the solid phase. Results are represented on Figure 4. Usually the starting temperature $\mathrm{T}_{\mathrm{B}}$ is chosen so that half of the enantiomeric excess is dissolved.

Table 1. Experimental conditions applied to the AS3PC tests and results.

\begin{tabular}{|c|c|c|c|c|c|c|c|c|c|c|}
\hline Run & $\begin{array}{c}( \pm) \\
\text { mass } \\
(\mathrm{g})\end{array}$ & $\begin{array}{c}\text { Solvent } \\
\text { mass }(g)\end{array}$ & $\begin{array}{l}\text { Initial } \\
\text { e.e. } \%\end{array}$ & \begin{tabular}{|c|}
$\mathbf{T}_{\mathbf{B}}$ \\
Starting \\
temp. $\left({ }^{\circ} \mathbf{C}\right)$
\end{tabular} & $\left|\begin{array}{cc}\mathbf{T}_{\mathrm{F}} & \text { Final } \\
\text { temp. } & \left({ }^{\circ} \mathbf{C}\right)\end{array}\right|$ & $\begin{array}{l}\text { Cooling } \\
\text { rate } \\
(\% / \mathrm{min})\end{array}$ & $\begin{array}{c}\text { Crude } \\
\text { crops } \\
\text { mass (g) }\end{array}$ & $\begin{array}{c}\text { e.e. } \\
\text { purity } \\
(\%)\end{array}$ & $\begin{array}{c}\text { Pure } \\
\text { enantiomer } \\
\text { mass }(g)\end{array}$ & $\begin{array}{l}\text { e.e. fmax } \\
(\%)\end{array}$ \\
\hline 1 & 4,375 & 30,625 & 10,0 & 43,0 & 25,0 & 0,3 & 0,596 & 61,5 & 0,481 & $5,2^{(*)}$ \\
\hline 2 & 4,375 & 30,625 & 6,0 & 41,5 & 25,0 & 0,5 & 0,948 & 44,3 & 0,684 & $7,3^{(\circ)}$ \\
\hline 3 & 4,988 & 34,916 & 3,8 & 41,0 & 26,5 & 0,5 & 0,608 & 69,0 & 0,514 & $4,9^{(0)}$ \\
\hline 4 & 4,375 & 30,625 & 4,7 & 41,0 & 27,0 & 0,8 & 0,594 & 65,3 & 0,491 & $5,3^{(\circ)}$ \\
\hline
\end{tabular}

\footnotetext{
(*): Magnetic stirring.

${ }^{(\circ)}$ : Smooth stirring with a blade.
} 
Four tests of preferential crystallization (AS3PC mode) were carried out. The experimental conditions and the corresponding results are collected in Table 1. Owing to the poor crystallization kinetics the first mixtures were cooled down to temperatures $\left(\mathrm{T}_{\mathrm{F}}\right)$ lower than that initially expected $\left(26.5^{\circ} \mathrm{C}\right)$. The cooling was stopped at $\mathrm{T}_{\mathrm{F}}$ which implies isothermal conditions in the second halves of the crystallization operations. For each test the course of the preferential crystallization was monitored by direct polarimetric measurements of the mother liquor (cf. Figure 5).

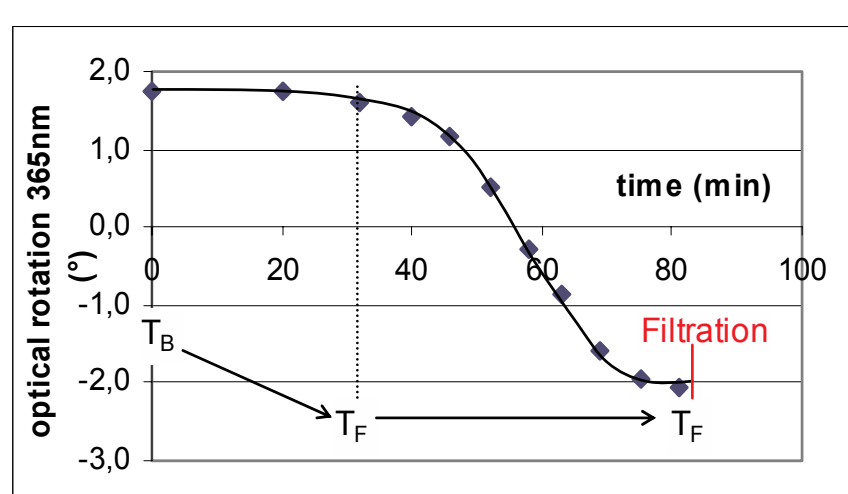

Figure 5. Mother liquor optical rotation vs. time during the entrainment $n^{\circ} 2$ of the title compound.
- Up to 40 minutes, the optical rotation only shows a small decrease: the supersaturation is insufficient to ensure the growth of the seeds and there is no enantioselective secondary nucleation.

- From 40 to 75 minutes, the inversion of the optical rotation clearly shows the entrainment effect: the system is out of equilibrium and the mother liquor is continuously enriching in the opposite enantiomer.

- After 75 minutes, the end of the optical rotation decrease is likely to be associated with the nucleation of the opposite enantiomer; the mixture must be swiftly filtered.

These first tests demonstrate the existence of the entrainment effect. Nevertheless, the efficiency remains low (e.e.fmax $5-7 \%$ ) i.e. the ethanolamine salt of $( \pm$ ) mandelic acid is another example of stable conglomerate which is associated with a poor entrainment effect [2]. Further analyses were carried out to determine the origin of such a poor efficiency.

\section{DISCUSSION AND ADDITIONAL WORKS}

Many phenomena could be at the origin of a poor entrainment effect. Among those, the most commonly encountered are:

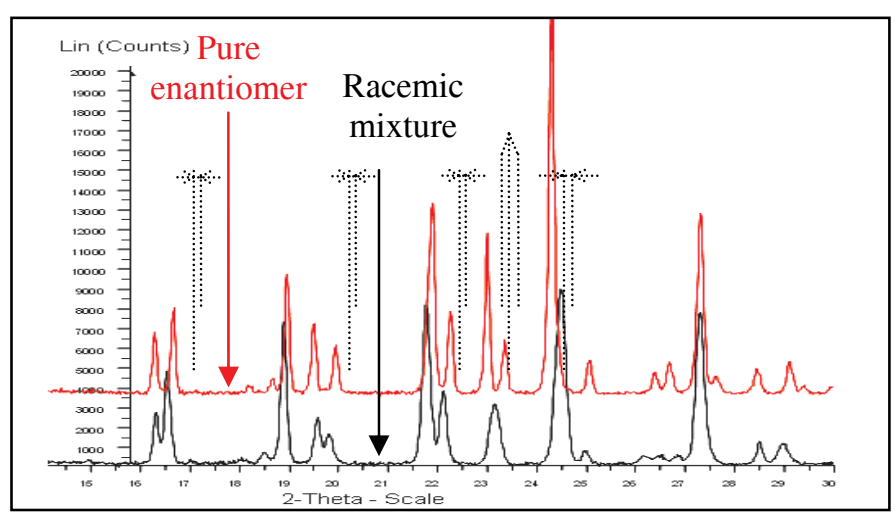

Figure 6. Zooms on accurate comparative XRPD analyses of the racemic mixture and the pure enantiomer.
- The existence of a metastable racemic compound $[2,3]$,

- Irreversible docking of the counter enantiomer [1],

- Lamellar epitaxy phenomena [1],

- The existence of a partial solid solution...

Precise XRPD analyses $\left(0.002^{\circ}\right.$ per step, 30s exposure per step, D5005 Siemens) on the racemic mixture and the pure enantiomer performed with internal standard (cf. Figure 6) 
revealed small by significant $2 \mathrm{~T}$ shifts between related peaks. Consistently, investigations in the (+) - (-) binary diagram by using DSC measurements together with hot stage microscopy (cf. Figure 7, only half of the phase diagram is represented) reveals that symmetrical partial solid solutions exist. This representation allows to estimate their maximum composition near $36 \%$ e.e. at $106^{\circ} \mathrm{C}$.

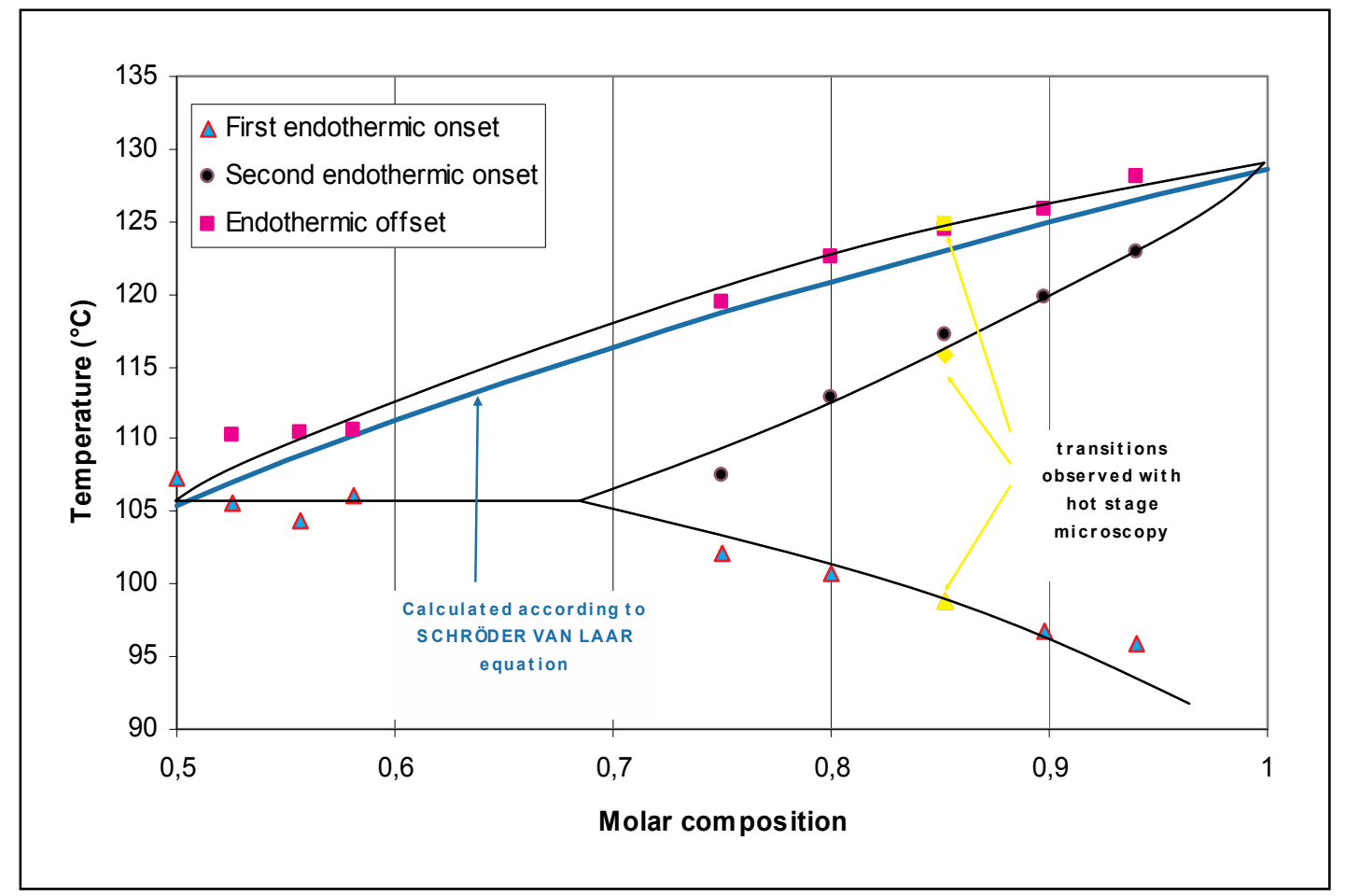

Figure 7. Half of symmetrical binary diagram of the (+) and (-) ethanolamine mandelate.

As the width of the domain of solid solution seems to diminish steeply with temperature (e.e. $>80 \%$ for temperature lower than $95^{\circ} \mathrm{C}$ so probably e.e. $>90 \%$ near room temperature), this diagram is not sufficient to explain the purity of the crude crops $(<70 \%$ e.e.). Nevertheless at least two hypotheses (not excluding each other) are likely to justify the poor performances of the preferential crystallization:

First hypothesis: (cf. Figure 8)

As the temperature is decreased, the point representative of the overall synthetic mixture enters into the three phase domain (o). The metastable equilibria involved in the AS3PC process clearly show that the solid phase has a lower enantiomeric purity than that at the equilibrium state (tie lines represented with dashed lines connect the compositions of the liquid phase (y) and the solid phase (x)).

Second hypothesis:

As the crystallization kinetics of the enantiomer in the presence of its antipode appears to be poor, the temperature versus time law has been, on purpose, made steeply. As a consequence, supersaturations may be high enough to prematurely induce the spontaneous nucleation of the opposite solid solution ( $E \mid 2.3)$. 


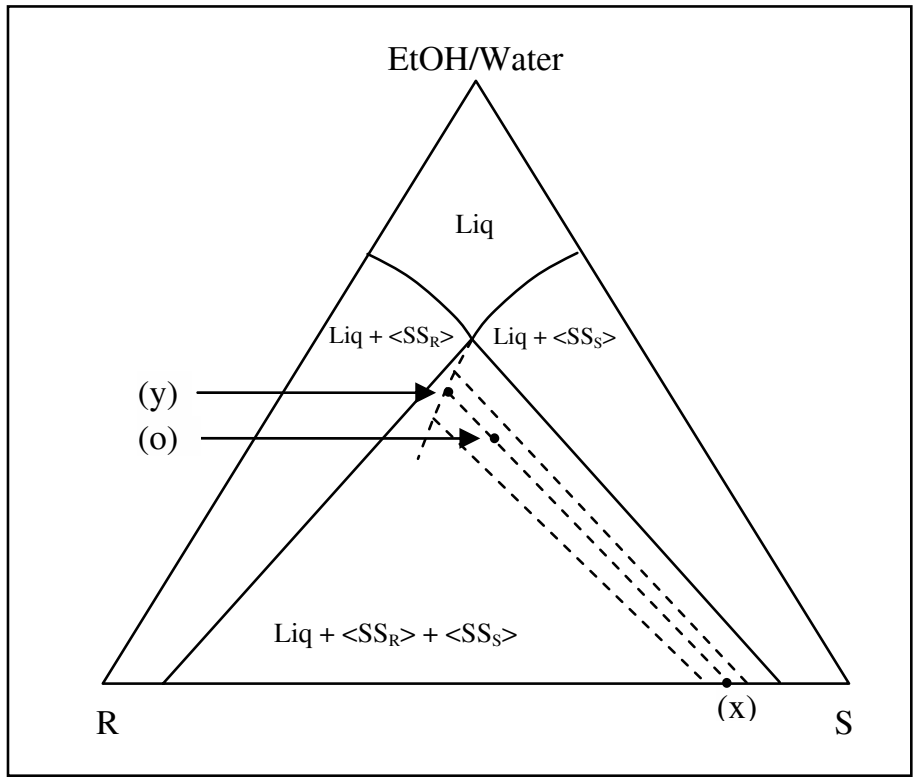

Figure 8. Isothermal ternary diagram - metastable equilibria represented with dashed lines.

\section{CONCLUSION}

Mixtures of (+) and (-) ethanolamine mandelates crystallize as a stable conglomerate but clear evidence of partial solid solutions between the enantiomers are given by using accurate XRPD and DSC analyses. The rather poor results of the preferential crystallization are consistent with this partial miscibility at the solid state only if metastable equilibria enlarging the monophasic solid state domains are considered and/or the high supersaturation necessary to induce an entrainment effect is above the threshold of primary nucleation of the second enantiomer. Future studies will be devoted to assess these two non contradictory hypotheses.

\section{References}

[1] Courvoisier L., Mignot L., Petit M.N. and Coquerel G., Org. Proc. Res. Dev., 7 (2003) 1007

[2] Dufour F., Perez G. and Coquerel G., Bull. Chem. Soc. Japan, 77 (2004) 79

[3] Druot-Houllemare S. and Coquerel G., J. Chem. Soc., Perkin Trans. 2, 10 (1998) 2211

[4] Coquerel G. and Perez G., J. Crystal Growth, 88 (1988) 511

[5] Gervais C., Beilles S., Cardinale P., Petit S. and Coquerel G., J. Phys. Chem., 106 (2002) 646 